\title{
THE STABILITY CONDITIONS OF FINITE DIFFERENCE SCHEMES FOR SCHRÖDINGER, KURAMOTO-TSZUKI AND HEAT EQUATIONS
}

\author{
M. RADŽIŪNAS, F. IVANAUSKAS \\ Weierstrass Institute for Applied Analysis and Stochastics \\ Mohrenstrasse 39, D - 10117 Berlin, Germany \\ Faculty of Mathematics at Vilnius University, \\ Naugarduko 24, LT-2600 Vilnius, Lithuania \\ E-mail: radziunas@wias-berlin.de Feliksas.Ivanauskas@maf.vu.lt
}

\begin{abstract}
We consider various finite difference schemes for the first and the second initial-boundary value problems for linear Kuramoto-Tsuzuki, heat and Schrödinger equations in $d$-dimensional case. Using spectral methods, we find the conditions of stability on initial data in the $L_{2}$ norm.
\end{abstract}

\section{INTRODUCTION}

There are many studies on the finite difference schemes for initial-boundary linear and nonlinear evolutionary problems. There are two-layered schemes with weights [4], [6]-[8], various three-layered schemes with and without weights [1]-[3], [7]-[9], also various splitting schemes [4], [7], [10], which allow to solve multidimensional problems using local one-dimensional schemes.

The purpose of the present paper is linear stability analysis of KuramotoTsuzuki, heat and Schrödinger equations. It is an important feature in the theory of nonlinear equations. It is difficult to expect a stability of the scheme for nonlinear equation, if the scheme for a linearized equation is unstable. An object of investigation is finite difference schemes approximating an equation

$$
\frac{\partial u}{\partial t}=(a+i b) \sum_{k=1}^{d} \frac{\partial^{2} u}{\partial x_{k}^{2}} .
$$

Here we have three cases of equations: 
1. If $a>0$ and $b=0$ we have a heat equation;

2. If $a>0$ and $b \neq 0$ we have the Kuramoto-Tsuzuki equation;

3 . If $a=0$ and $b \neq 0$ we have the Schrödinger equation.

In the present paper we are investigating how the condition of stability of difference schemes (Courant condition) depends on the coefficients $a$ and $b$ and other parameters of finite difference schemes. As a rule there are no connection between heat and Schrödinger equations in numerical analysis. In the present paper an attempt was made to unite these equations into one class continuously varying the coefficients $a$ and $b$. The unification is made considering finite difference schemes for these equations.

In $\S 4-\S 6$ we have investigated and found the stability conditions in the norm of space $L_{2}$ for two-layered weighted finite difference schemes and two types of weighted splitting schemes. In $\S 7-\S 9$ we have found the conditions on parameters, when necessary von Neumann's stability condition holds for a hopscotch difference scheme, DuFort-Frankel type and other three-layered weighted schemes. We have used spectral methods [5], [7] in stability analysis. Note that the stability conditions are the same for both first and the second boundary value problems.

All the results of analysis are presented in a table at the end of the paper.

\section{FORMULATION OF THE PROBLEM. MAIN NOTATIONS}

We consider some finite difference schemes for the linear Schrödinger, Kuramoto-Tsuzuki or a heat equation:

$$
\frac{\partial u}{\partial t}=(a+i b) \Delta u, \quad(\mathbf{x}, t) \in \bar{Q} .
$$

Here $\mathbf{x}=x_{1}, \ldots, x_{d} ; u(\mathbf{x}, t)$ is a complex-valued function; $i=\sqrt{-1} ; \Delta$ is $d$ dimensional Laplacian; $a \geq 0, a^{2}+b^{2} \neq 0 ; \bar{\Omega}=[0,1]^{d}$ is $d$-dimensional domain with a boundary $\Gamma=\partial \bar{\Omega} ; \bar{Q}=\Omega \times[0, T]$ is $(d+1)$-dimensional domain with a boundary $S=\Gamma \times[0, T]$. Let us consider an initial condition

$$
u(\mathbf{x}, 0)=u_{0}(\mathbf{x}), \quad \mathbf{x} \in \bar{\Omega}
$$

and boundary conditions of the first or second type

$$
u(\mathbf{x}, t)=0, \quad \text { or } \quad \frac{\partial u}{\partial \mathbf{n}}(\mathbf{x}, t)=0, \quad(\mathbf{x}, t) \in S .
$$

Here $\mathbf{n}$ is normal vector to the boundary $S$ of the domain $\bar{Q}$.

Let us denote the uniform grid with the steps $\tau$ and $h_{j}$ in the domain $\bar{Q}$ and the following notations in this grid:

$$
\tau=T / M, \quad h_{j}=1 / N_{j} \quad j=1, \ldots, d, \quad h=\min \left\{h_{1}, \ldots, h_{d}\right\},
$$




$$
\begin{aligned}
\bar{h}= & \max \left\{h_{1}, \ldots, h_{d}\right\} ; \bar{Q}_{h}=\bar{\omega}_{h} \times \bar{\omega}_{\tau}, \quad \tilde{Q}_{h}=\bar{\omega}_{h} \times \omega_{\tau}, \quad Q_{h}=\omega_{h} \times \omega_{\tau} ; \\
t_{j}= & j \tau, \quad \bar{\omega}_{\tau}=\left\{t_{j} ; j=0, \ldots, M\right\}, \omega_{\tau}=\left\{t_{j}=\tau j ; j=0, \ldots, M-1\right\} \\
& \text { or } \omega_{\tau}=\left\{t_{j} ; j=1, \ldots, M-1\right\} \quad \text { for three }- \text { layered schemes; } \\
x_{l, r_{l}}= & r_{l} h_{l}, \quad \bar{\omega}_{h}=\bar{\omega}_{1, h} \times \ldots \times \bar{\omega}_{d, h}, \quad \omega_{h}=\omega_{1, h} \times \ldots \times \omega_{d, h} \\
\bar{\omega}_{l, h}= & \left\{x_{l, r_{l}}, r_{l}=0, \ldots, N_{l}\right\}, \quad \omega_{l, h}=\left\{x_{l, r_{l}}, r_{l}=1, \ldots, N_{l}-1\right\} .
\end{aligned}
$$

For the second boundary value problem we introduce also the fictitious grid points $\left(x_{1, r_{1}}, \ldots, x_{l,-1}, \ldots, x_{d, r_{d}}\right)$ and $\left(x_{1, r_{1}}, \ldots, x_{l, N_{l}+1}, \ldots, x_{d, r_{d}}\right)$, where $r_{l}=0, \ldots, N_{l}, x_{l,-1}=-h_{l}, x_{l, N_{l}+1}=1+h_{l}$.

Let function $f=f\left(x_{l}\right)$ be some grid function on a grid $\bar{\omega}_{l, h}$. Let

$$
S_{l} f=0.5 h_{l}(f(0)+f(1))+\sum_{x_{l} \in \omega_{l, h}} f\left(x_{l}\right) h_{l} .
$$

Let us define an inner product and the norm of grid functions $u$ and $v$ in a space $L_{2 h}$, the grid analogue of the space $L_{2}$, as

$$
(u, v)=S_{1}\left(S_{2}\left(\cdots S_{d}\left(u(\mathbf{x}) v^{*}(\mathbf{x})\right) \cdots\right)\right), \quad\|u\|=\sqrt{(u, u)} .
$$

We use the following notations for the grid functions:

$$
\begin{aligned}
p & =p_{\mathbf{r}}^{j}=p\left(x_{1, r_{1}}, \ldots, x_{d, r_{d}}, t_{j}\right), \quad \hat{p}=p_{\mathbf{r}}^{j+1}, \quad \check{p}=p_{\mathbf{r}}^{j-1}, \quad p_{t}=(\hat{p}-p) / \tau \\
T_{l}^{-} p & =p\left(x_{1, r_{1}}, \ldots, x_{l, r_{l}-1}, \ldots, x_{d, r_{d}}, t_{j}\right), T_{l}^{+} p=p\left(x_{1, r_{1}}, \ldots, x_{l, r_{l}+1}, \ldots, x_{d, r_{d}}, t_{j}\right), \\
p_{x_{l}} & =\left(T_{l}^{+} p-p\right) / h_{l}, \quad p_{\bar{x}_{l}}=\left(p-T_{l}^{-} p\right) / h_{l}, \quad p_{\dot{x}_{l}}=\left(T_{l}^{+} p-T_{l}^{-} p\right) / 2 h_{l}, \\
p_{\bar{x}_{l} x_{l}} & =\Delta_{h, l} p=\left(p_{x_{l}}-p_{\bar{x}_{l}}\right) / h_{l}, \quad \Delta_{h} p=\sum_{l=1}^{d} p_{\bar{x}_{l} x_{l}}
\end{aligned}
$$

Investigating properties of finite difference schemes, in all cases we consider initial conditions

$$
p(\mathbf{x}, 0)=u_{0}(\mathbf{x}), \quad \mathbf{x} \in \bar{\omega}_{h}
$$

and zero boundary conditions for the first boundary value problem

$$
p(\mathbf{x}, t)=0, \quad(\mathbf{x}, t) \in S \cap \bar{Q}_{h},
$$

or grid analogue of the Neumann condition for the second boundary problem

$$
p_{\dot{x}_{l}}(\mathbf{x}, t)=0, \quad(\mathbf{x}, t) \in\left(x_{l}=0 \cup x_{l}=1\right) \cap \bar{Q}_{h} .
$$

In the case of the first boundary value problem we shall define

$$
v_{\mathbf{k}}=\prod_{l=1}^{d} v_{k_{l}}, \quad v_{k_{l}}=\sqrt{2} \sin \left(\pi k_{l} x_{l}\right), \text { here } k_{l}=1, \ldots, N_{l}-1
$$


For the second boundary value problem we shall use similar notations

$$
v_{\mathbf{k}}=\prod_{l=1}^{d} v_{k_{l}}, \quad v_{k_{l}}= \begin{cases}\cos \left(\pi k_{l} x_{l}\right)=1, & \text { if } k_{l}=0 \\ \sqrt{2} \cos \left(\pi k_{l} x_{l}\right), & \text { if } k_{l}=1, \ldots, N_{l}-1 \\ \cos \left(\pi k_{l} x_{l}\right), & \text { if } k_{l}=N_{l}\end{cases}
$$

These systems are complete orthonormal systems of grid functions in the space $L_{2 h}$ with zero values or zero derivative values on the boundary. For the simplicity, for some fixed vector $\mathbf{k}$ we shall often denote the functions from these systems as $v=v_{\mathbf{k}}$ and $w=v_{\mathbf{N}-\mathbf{k}}$.

We shall note also that for the both boundary value problems a set of functions $v_{\mathbf{k}}$ is a complete system of orthogonal eigenfunctions of the problem

$$
\Delta_{h} X(\mathbf{x})+\lambda X(\mathbf{x})=0, \quad \mathbf{x} \in \omega_{h} \quad\left(\text { or } \quad \mathbf{x} \in \bar{\omega}_{h} \text { in the second case }\right)
$$

with the first (2.5) or the second (2.6) boundary conditions on the grid.

All eigenvalues of the first and the second boundary problem are in the corresponding interval:

$$
\lambda \in\left[\sum_{l=1}^{d} \frac{4}{h_{l}^{2}} \sin ^{2} \frac{\pi h_{l}}{2} ; \sum_{l=1}^{d} \frac{4}{h_{l}^{2}} \cos ^{2} \frac{\pi h_{l}}{2}\right], \quad \text { or } \quad \lambda \in\left[0 ; \sum_{l=1}^{d} \frac{4}{h_{l}^{2}}\right] .
$$

Investigating splitting schemes we shall also use the notation $\lambda^{(l)}$ for the eigenvalues of the locally one dimensional problem

$$
\Delta_{h, l} X\left(x_{l}\right)+\lambda^{(l)} X\left(x_{l}\right)=0, \quad x_{l} \in \omega_{h, l} \quad \text { (or } x_{l} \in \bar{\omega}_{h, l} \text { in the second case) }
$$

with corresponding boundary conditions. The values of $\lambda^{(l)}$ can be described in the same way as in (2.9) taking $d=1$.

Let us also introduce some notations, which we shall use extensively investigating difference schemes below in this paper:

$$
\gamma=a+i b, \quad \nu_{l}=\frac{2 \tau}{h_{l}^{2}}, \quad \nu=\sum_{l=1}^{d} \nu_{l}, \quad \delta^{(l)}=\frac{\lambda^{(l)} \tau}{2 \nu_{l}} \in[0 ; 1], \quad \delta=\frac{\lambda \tau}{2 \nu} \in[0 ; 1] .
$$

\section{GENERAL STABILITY REQUIREMENTS}

In the next sections we shall investigate two or three-layered finite difference schemes. These schemes we can write as the following equations:

$$
\bar{A}_{1} \hat{p}=\bar{A}_{2} p+\bar{A}_{3} \check{p} \equiv \hat{p}=\bar{A}_{1}^{-1} \bar{A}_{2} p+\bar{A}_{1}^{-1} \bar{A}_{3} \check{p}=A_{2} p+A_{3} \check{p} .
$$


$\bar{A}_{1}, \bar{A}_{2}, \bar{A}_{3}$ are linear operators in the space $\mathbf{B}, \bar{A}_{1}$ is non-degenerate operator.

Taking into account boundary conditions (2.5) or (2.6), we consider $\mathbf{B}=$ $L_{2 h}$ for two-layered schemes, $\mathbf{B}=L_{2 h} \times L_{2 h}$ for three-layered schemes. Let $A: \mathbf{B} \rightarrow \mathbf{B}$ be a linear amplification operator:

$$
\hat{p}=A p \quad \text { or } \quad\left(\begin{array}{l}
\hat{p} \\
p
\end{array}\right)=A\left(\begin{array}{c}
p \\
\check{p}
\end{array}\right) .
$$

In all our finite difference schemes this operator does not depend on time. Thus, for all $j=0, \ldots, M$ we can write

$$
p\left(t_{j}\right)=A^{j} p\left(t_{0}\right) \quad \text { or } \quad\left(\begin{array}{c}
p\left(t_{j+1}\right) \\
p\left(t_{j}\right)
\end{array}\right)=A^{j}\left(\begin{array}{c}
p\left(t_{1}\right) \\
p\left(t_{0}\right)
\end{array}\right)
$$

We shall use the following definitions of the stability of the difference schemes:

Definition 3.1. The difference scheme (3.1) is stable on initial data, if there are positive constants $\tau_{0}$ and $h_{l 0}$ such that for all $0<\tau \leq \tau_{0}$ and $0<h_{l} \leq h_{l 0}$ the inequalities

$$
\left\|A^{j}\right\| \leq \bar{C} \Rightarrow\left\|p\left(t_{j}\right)\right\| \leq \bar{C}\left\|p\left(t_{0}\right)\right\| \quad\left(\text { or }\left\|p\left(t_{j+1}\right)\right\| \leq \bar{C}\left(\left\|p\left(t_{0}\right)\right\|+\left\|p\left(t_{1}\right)\right\|\right)\right)
$$

hold. Here $j=0, \ldots, M$ and the constant $\bar{C}$ is independent from $\tau, h_{k}, t_{j}$.

Definition 3.2. (von Neumann's necessary stability condition) [6]. Let $\lambda_{A}$ be a set of all eigenvalues of the not time-dependent operator $A$. Let $\bar{\lambda}=\left|\lambda_{1}\right|=$ $\max \left\{\left|\lambda_{A}\right|\right\}$. Then the necessary stability condition of the difference scheme (3.1) reads as $\bar{\lambda} \leq 1+C \tau$, where $\tau \rightarrow 0$ and $C=\ln \bar{C} / T$.

In the case of two-layered weighted schemes, splitting schemes and hopscotch type schemes we have $\bar{A}_{3} \equiv 0$ and $A=A_{2}$. We shall find for these schemes that either grid functions $v_{\mathbf{k}}$ are the eigenfunctions of $A$, or functions $v_{\mathbf{k}}$ and $v_{\mathbf{N}-\mathbf{k}}$ compose a proper subspace of this operator. Thus, using the functions $v_{\mathbf{k}}$ as a basis of a space $\mathbf{B}$ and numbering functions $v_{\mathbf{k}}$ and $v_{\mathbf{N}-\mathbf{k}}$ as a neighbouring vectors, we can write the operator $A$ as a diagonal matrix or a matrix, decomposed into diagonal $2 \times 2$ blocks, which we denote as $A_{\mathbf{k}}$.

If $A$ is a diagonal matrix and $A(\mathbf{k}, \mathbf{k})=\lambda_{\mathbf{k}}$ are diagonal elements, the stability condition $\left\|A^{j}\right\| \leq \bar{C}$ is equivalent to the requirement $|A(\mathbf{k}, \mathbf{k})| \leq$ $1+C \tau$ for all possible $\mathbf{k}$. Thus, in this case both definitions are equivalent.

If $A$ is a blocked diagonal matrix then the condition $\left\|A^{j}\right\| \leq \bar{C}$ is equivalent to $\left\|A_{\mathbf{k}}^{j}\right\| \leq \bar{C}$ with the same constant $\bar{C}$ for all possible $\mathbf{k}$.

In the case of three-layered schemes we have

$$
A=\left(\begin{array}{cc}
A_{2} & A_{3} \\
I & 0
\end{array}\right)
$$


where $I$ is identity operator and 0 is zero operator. In all difference schemes we investigate, the functions $v_{\mathbf{k}}$ are the eigenfunctions of the operators $A_{2}$ and $A_{3}$. A set of functions $\left(v_{\mathbf{k}}, 0\right)^{T}$ and $\left(0, v_{\mathbf{k}}\right)^{T}$ is a complete orthonormal system of a space $\mathbf{B}=L_{2 h} \times L_{2 h}$. Here $(u, v)$ is a row vector and $(u, v)^{T}$ is a column vector. Enumerating the basis of the space $\mathbf{B}$ in a such way that the functions $\left(v_{\mathbf{k}}, 0\right)^{T}$ and $\left(0, v_{\mathbf{k}}\right)^{T}$ be a neighbouring functions, we can write the matrix of the operator $A$ as blocked diagonal matrix with $2 \times 2$ blocks on a diagonal which we denote as $A_{\mathbf{k}}$. Here

$$
A_{\mathbf{k}}=\left(\begin{array}{cc}
A_{2}(\mathbf{k}, \mathbf{k}) & A_{3}(\mathbf{k}, \mathbf{k}) \\
1 & 0
\end{array}\right) .
$$

Analogously as for the blocked diagonal matrices $A$ for two-layered schemes, a stability condition $\left\|A^{j}\right\| \leq \bar{C}$ is equivalent to the condition $\left\|A_{\mathbf{k}}^{j}\right\| \leq \bar{C}$ with the same constant $\bar{C}$ for all possible $\mathbf{k}$.

\section{TWO-LAYERED WEIGHTED DIFFERENCE SCHEME}

We consider a two-layered finite difference scheme with a weight $\sigma \in[0, \infty)$ :

$$
\frac{\hat{p}-p}{\tau}=(a+i b) \Delta_{h}(\sigma \hat{p}+(1-\sigma) p), \quad(\mathbf{x}, t) \in Q_{h}\left(\text { or } \tilde{Q}_{h}\right) .
$$

Here and for all other difference schemes below we suppose, that the solution $u(\mathbf{x}, t)$ is smooth enough, $\sigma$ is not dependent on the grid steps and the conditions (2.4) and (2.5) (or (2.6)) are fulfilled.

We can write the difference scheme (4.1) as

$$
\hat{p}=\left(I-\gamma \tau \sigma \Delta_{h}\right)^{-1}\left(I+\gamma \tau(1-\sigma) \Delta_{h}\right) p=A p .
$$

Since $a \geq 0$, an operator $\left(I-\gamma \tau \sigma \Delta_{h}\right)$ is non-degenerate, and all the functions $v=v_{\mathbf{k}}$ from (2.7) or (2.8) are eigenfunctions of the operator $A$. We can write the following equalities for the eigenvalues of the scheme:

$$
q=\frac{1-2 \nu \delta \gamma(1-\sigma)}{1+2 \nu \delta \gamma \sigma} \equiv|q|^{2}-1=4 \frac{\nu^{2} \delta^{2}|\gamma|^{2}(1-2 \sigma)-a \nu \delta}{(1+2 a \nu \delta \sigma)^{2}+(2 b \nu \delta \sigma)^{2}}
$$

Note that $|q| \leq 1+C \tau \equiv|q|^{2}-1 \leq C \tau$ when $\tau \rightarrow 0$. Thus, we require

$$
\nu^{2} \delta^{2}|\gamma|^{2}(1-2 \sigma)-a \nu \delta \leq C \tau\left((1+2 a \nu \delta \sigma)^{2}+(2 b \nu \delta \sigma)^{2}\right) .
$$

If $\sigma \in[0.5, \infty)$, this inequality is satisfied unconditionally for both boundary value problems.

Let $\sigma \in[0,0.5)$, then $\nu^{2} \delta^{2}|\gamma|^{2}(1-2 \sigma)>0$. Let us consider the value of $|q|^{2}-1$ as a function dependent on $\nu \delta$. Note that $\nu \delta \in[0 ; \nu]$. When $\nu \delta$ 
increases from 0 till some value $\overline{\nu \delta},|q|^{2}-1$ decreases from 0 till some negative value. When $(\nu \delta) \rightarrow \infty,|q|^{2}-1$ increases till $(1-2 \sigma) / \sigma^{2}>C \tau>0$. Thus, the value of $\nu$ must be bounded. Now the stability requirement is equivalent to

$\nu^{2}|\gamma|^{2}(1-2 \sigma)-a \nu \leq C \tau\left((1+2 a \nu \sigma)^{2}+(2 b \nu \sigma)^{2}\right) \equiv \nu|\gamma|^{2}(1-2 \sigma)-2 a \leq C \tau / \nu$.

From here we directly obtain the stability requirements for the difference scheme and complete the proof of the following theorem:

THEOREM 4.1. Suppose that the solution of the differential problem is smooth enough. Then if $\sigma \in[0.5, \infty)$, the difference scheme (4.1),(2.4),(2.5) (or (2.6)) is unconditionally stable for the evolutionary equation of any type.

If $\sigma \in[0,0.5)$ then this scheme is stable when the following conditions hold for the Kuramoto-Tsuzuki, heat and Schrödinger equations respectively:

$$
\sum_{k=1}^{d} \frac{2 \tau}{h_{k}^{2}} \leq \frac{a}{\left(a^{2}+b^{2}\right)(1-2 \sigma)}+C h^{2}, \quad \sum_{k=1}^{d} \frac{2 \tau}{h_{k}^{2}} \leq \frac{1}{a(1-2 \sigma)}+C h^{2}, \quad \sum_{k=1}^{d} \frac{\tau}{h_{k}^{4}} \leq C .
$$

\section{SPLITTING SCHEME I}

Let $d \geq 2$. We consider the following splitting finite difference scheme with weights $\sigma_{l} \in[0, \infty), l=1,2, \ldots, d$ :

$$
\begin{aligned}
\frac{p^{(l)}-p^{(l-1)}}{\tau} & =(a+i b)\left(\sigma_{k} p^{(l)}+\left(1-\sigma_{l}\right) p^{(l-1)}\right)_{\bar{x}_{l} x_{l}}, \quad(\mathbf{x}, t) \in Q_{h}\left(\text { or } \tilde{Q}_{h}\right) \\
l & =1, \ldots, d, \quad p^{(0)}=p, \quad p^{(d)}=\hat{p} .
\end{aligned}
$$

We can write the equations of a scheme (5.1) as

$$
p^{(l)}=\left(I-\gamma \tau \sigma_{l} \Delta_{h, l}\right)^{-1}\left(I+\gamma \tau\left(1-\sigma_{l}\right) \Delta_{h, l}\right) p^{(l-1)}=A_{l} p^{(l-1)} .
$$

Similarly as in $\S 4$ we note that the functions $v_{\mathbf{k}}$ from (2.7) or (2.8) are eigenfunctions of the operators $A_{l}, l=1, \ldots, d$ and of operator $A=A_{d} A_{d-1} \cdots A_{1}$, where $\hat{p}=A p$. Note, that $v, v^{(l)}, \hat{v}$ satisfy boundary conditions. We have

$$
\hat{v}=\prod_{l=1}^{d} q^{(l)} v=q v, \quad q^{(l)}=\frac{1-\lambda^{(l)} \tau \gamma\left(1-\sigma_{l}\right)}{1+\lambda^{(l)} \tau \gamma \sigma_{l}}=\frac{1-2 \nu_{l} \delta^{(l)} \gamma\left(1-\sigma_{l}\right)}{1+2 \nu_{l} \delta^{(l)} \gamma \sigma_{l}} .
$$

THEOREM 5.1. Suppose that the solution of the differential problem is smooth enough. Then if for any $l=1, \ldots, d$ the weight $\sigma_{l} \in[0.5, \infty)$, the difference scheme (5.1),(2.4),(2.5) (or (2.6)) is unconditionally stable for the evolutionary equation of any type. 
If there are such indexes $l$ that $\sigma_{l} \in[0,0.5)$ then this scheme is stable when for all these indexes the following conditions hold for the Kuramoto-Tsuzuki, heat and Schrödinger equations respectively:

$$
\frac{2 \tau}{h_{l}^{2}} \leq \frac{a}{\left(a^{2}+b^{2}\right)\left(1-2 \sigma_{l}\right)}+C h_{l}^{2}, \quad \frac{2 \tau}{h_{l}^{2}} \leq \frac{1}{a\left(1-2 \sigma_{l}\right)}+C h_{l}^{2}, \quad \frac{\tau}{h_{l}^{4}} \leq C .
$$

Proof. The first part of this theorem follows from the estimates $\left|q^{(l)}\right| \leq 1$ if only $\sigma_{l} \in[0.5, \infty)$.

We see, that the requirements at the second part of the theorem are sufficient to achieve the estimate $|q| \leq 1+C \tau$. These requirements are necessary also. On contrary, assume that there is $l$ such that $\sigma_{l} \in[0,0.5)$ and the restriction on $\tau$ and $h_{l}$ is not satisfied. For the simplicity, $l=1$. Then for the first or the second boundary problem we respectively consider the eigenfunctions

$v=\left(\sqrt{2}^{d} \sin \left(\pi\left(N_{1}-1\right) x_{1}\right) \times \sin \left(\pi x_{2}\right) \times \cdots \times \sin \left(\pi x_{d}\right)\right.$ and $v=\cos \left(\pi N_{1} x_{1}\right)$.

Using the results of $\S 4$ we can easily show that for all $\tau$ small enough we have $\left.\left|\prod_{l=2}^{d}\right| q^{(l)}\right|^{2}-1 \mid \leq C \tau$ and $|q|^{2} \geq(1-C \tau)\left|q^{(1)}\right|^{2}$. Since we suppose that difference scheme is stable, we have $|q|^{2} \leq 1+C^{\prime} \tau$ and from here it follows the estimate $\left|q^{(1)}\right|^{2} \leq 1+C^{\prime \prime} \tau$. Due to the Theorem 1 , this contradict to the supposition that there is no restrictions on $\tau$ and $h_{1}$. This completes the proof of this theorem.

\section{SPLITTING SCHEME II}

Let $d \geq 2$. We consider the following splitting finite difference scheme with weights $\sigma_{l} \in[0, \infty), l=1,2, \ldots, d$ :

$$
\begin{aligned}
\alpha_{l} \frac{p^{(l)}-p}{\tau} & =(a+i b)\left(\sigma_{l} p^{(l)}+\left(1-\sigma_{l}\right) p\right)_{\bar{x}_{l} x_{l}}, \quad(\mathbf{x}, t) \in Q_{h}, \quad\left(\text { or } \tilde{Q}_{h}\right), \\
l & =1, \ldots, d, \quad \alpha_{l}>0, \quad \sum_{l=1}^{d} \alpha_{l}=1, \quad \sum_{l=1}^{d} \alpha_{l} p^{(l)}=\hat{p} .
\end{aligned}
$$

In addition to $(2.10)$ for all $l=1, \ldots, d$ we denote

$$
\eta_{l}=\frac{\alpha_{l}}{\sigma_{l}}, \quad y_{l}=\frac{\tau \lambda^{(l)}}{\eta_{l}}=\frac{2 \nu_{l} \delta^{(l)}}{\eta_{l}}, \quad \varphi_{l}=|\gamma|^{2} y_{l}^{2}+2 a y_{l}+1, \quad \varepsilon=\sum_{l=1}^{d} \eta_{l}-2 .
$$

Note that for both boundary value problems we can consider $y_{l} \in\left[0 ; 2 \nu_{l} / \eta_{l}\right]$.

We can write the equations of a scheme (6.1) as

$$
p^{(l)}=\left(\alpha_{l} I-\gamma \tau \sigma_{l} \Delta_{h, l}\right)^{-1}\left(\alpha_{l} I+\gamma \tau\left(1-\sigma_{l}\right) \Delta_{h, l}\right) p=A_{l} p .
$$


The functions $v_{\mathbf{l}}$ from (2.7) or (2.8) are eigenfunctions of the operators $A_{l}$, $l=1, \ldots, d$ and the operator $A=\sum_{l=1}^{d} \alpha_{l} A_{l}$, where $\hat{p}=A p$. Note, that $v$, $v^{(l)}, \hat{v}$ satisfy the boundary conditions. We find

$$
v^{(l)}=q^{(l)} v=\frac{1-\lambda^{(l)} \tau \gamma\left(1-\sigma_{l}\right) / \alpha_{l}}{1+\lambda^{(l)} \tau \gamma \sigma_{l} / \alpha_{l}} v=\left(\left(1-\frac{1}{\sigma_{l}}+\frac{a y_{l}+1}{\sigma_{l} \varphi_{l}}\right)-i \frac{b y_{l}}{\sigma_{l} \varphi_{l}}\right) v .
$$

The eigenvalue $q$ of the operator $A$ is

$$
q=\sum_{l=1}^{d} \alpha_{l} q^{(l)}=\left(-1-\varepsilon+\sum_{l=1}^{d} \eta_{l} \frac{a y_{l}+1}{\varphi_{l}}\right)-i \sum_{l=1}^{d} \eta_{l} \frac{b y_{l}}{\varphi_{l}} .
$$

Using the expression of $\varepsilon$ we can find the value of $|q|^{2}-1$.

$$
\begin{aligned}
|q|^{2}-1 & =\left(-1-\varepsilon+\sum_{l=1}^{d} \eta_{l} \frac{a y_{l}+1}{\varphi_{l}}\right)^{2}+\left(\sum_{l=1}^{d} \eta_{l} \frac{b y_{l}}{\varphi_{l}}\right)^{2}-1 \\
& =\sum_{l=1}^{d} \frac{\eta_{l}\left(\varepsilon|\gamma|^{2} y_{l}^{2}-2 a y_{l}\right)}{\varphi_{l}}-\sum_{k<l} \frac{\eta_{k} \eta_{l}|\gamma|^{2}\left(y_{k}-y_{l}\right)^{2}}{\varphi_{k} \varphi_{l}} .
\end{aligned}
$$

THEOREM 6.1. Suppose that the solution of the differential problem is smooth enough. Then the inequality $\varepsilon=\sum_{l=1}^{d} \frac{\alpha_{l}}{\sigma_{l}}-2 \leq 0$ for the weights of the difference scheme (6.1),(2.4),(2.5) (or (2.6)) is necessary and sufficient estimate for the unconditional stability of the scheme for the evolutionary equation of any type.

If $\varepsilon>0$, then the following restrictions on the time and spatial grid steps are sufficient stability conditions of the difference scheme for Kuramoto-Tsuzuki, heat and Schrödinger equations respectively:

$$
\frac{2 \tau}{h_{l}^{2}} \leq \frac{a \alpha_{l}}{\varepsilon\left(a^{2}+b^{2}\right) \sigma_{l}}+C h_{l}^{2}, \quad \frac{2 \tau}{h_{l}^{2}} \leq \frac{\alpha_{l}}{\varepsilon a \sigma_{l}}+C h_{l}^{2}, \quad \frac{\tau}{h_{l}^{4}} \leq C \text { for all } l=1, \ldots, d .
$$

Proof. Note that $a \geq 0$, for all $\tau, h_{l}, \lambda^{(l)}$ the values $y_{l} \geq 0$ and $\varphi_{l} \geq 1$. From the estimate $\varepsilon \leq 0$ the non-positiveness of the right-hand side of the last equality follows. Thus, for all $\mathbf{k}$ the eigenvalue $q_{\mathbf{k}}$ satisfies the estimate $\left|q_{\mathbf{k}}\right|^{2} \leq$ 1. Since operator $A$ can be written as diagonal matrix, this estimate indicates stability of the scheme. Thus, $\varepsilon \leq 0$ is sufficient estimate for unconditional stability of the scheme.

Now suppose, that our scheme is unconditionally stable. Since there are no restrictions on the grid steps $h_{l}$ and $\tau$, taking different values of these grid steps and different values of $\lambda^{(l)}$ we can vary $y_{l}$ in the interval $(0, \infty)$. It is also possible to select such $\lambda^{(l)}$ and $h_{l}$ that $y_{l}=y$ for all $l=1, \ldots, d$, and $y \rightarrow \infty$ when $\tau, \bar{h} \rightarrow 0$. In this case we have the following:

$$
|q|^{2}-1=\sum_{l=1}^{d} \frac{\eta_{l}\left(\varepsilon|\gamma|^{2} y^{2}-2 a y\right)}{\varphi_{l}} \rightarrow \varepsilon(2+\varepsilon)>C \tau, \quad \text { when } \quad y_{l}=y \rightarrow \infty .
$$


It is obvious, that if $\varepsilon>0$, the condition $|q|^{2} \leq 1+C \tau$ could not be satisfied in this case. Thus, the estimate $\varepsilon \leq 0$ is also necessary for unconditional stability of the scheme. Therefore, the first part of this theorem is proved.

In the case when $\varepsilon>0$ some restrictions on time and space grid steps $\tau$ and $h_{l}$ appear. Considering different sets of parameters $\alpha_{l}$ and $\sigma_{l}$ we have different cases of these restrictions. In any case a sufficient condition of the stability of difference schemes for all evolutionary equations is

$$
\varepsilon|\gamma|^{2} y_{l}^{2}-2 a y_{l} \leq C \tau \quad l=1, \ldots, d,
$$

since this condition leads to the estimate $|q|^{2} \leq 1+C^{\prime} \tau$. From here the proof of the second part of the theorem follows.

\section{HOPSCOTCH TYPE SCHEME}

We consider an explicit finite difference scheme on the grid $Q_{h}$ or $\tilde{Q}_{h}$ :

$$
\begin{aligned}
& 2 \frac{\tilde{p}_{e}-p_{e}}{\tau}=(a+i b) \Delta_{h} p_{e} \quad(a), \quad 2 \frac{\tilde{p}_{o}-p_{o}}{\tau}=(a+i b) \Delta_{h} \tilde{p}_{o} \quad(b), \\
& 2 \frac{\hat{p}_{o}-\tilde{p}_{o}}{\tau}=(a+i b) \Delta_{h} \tilde{p}_{o} \quad(c), \quad 2 \frac{\hat{p}_{e}-\tilde{p}_{e}}{\tau}=(a+i b) \Delta_{h} \hat{p}_{e} \quad(d) .
\end{aligned}
$$

Here $p_{e}=p\left(\mathbf{x}_{\mathbf{e}}\right),|\mathbf{e}|=e_{1}+e_{2}+\cdots+e_{d}$ is even number, $p_{o}=p\left(\mathbf{x}_{\mathbf{o}}\right),|\mathbf{o}|$ is odd number. In addition to (2.10) for the fixed $\mathbf{k}$ we define

$$
\bar{c}=\sum_{l=1}^{d} \nu_{l} \cos \left(\pi k_{l} h_{l}\right)=-\sum_{l=1}^{d} \nu_{l} \cos \left(\pi\left(N_{l}-k_{l}\right) h_{l}\right) \in[-\nu ; \nu] .
$$

Here $k_{l}=1, \ldots, N_{l}-1\left(0, \ldots, N_{l}\right)$ for the first (second) boundary problem.

We shall investigate how operator $A, A v=\hat{v}$, described in the equations (7.1), transforms function $v=v_{\mathbf{k}}$ from (2.7) or (2.8). Note that the coefficients $r_{j}$ and $r_{j}^{\prime}, j=1, \ldots, 4$, introduced below do not depend on the grid point $x$ but depend just on the vector number $\mathbf{k}$ of the function $v=v_{\mathbf{k}}$. Deriving the expressions from the $(7.1 \mathrm{~b}-\mathrm{d})$ we use the fact, that the neighbours of each 'odd' ('even') point are 'even' ('odd') points, and the function in these neighbouring points satisfies the formulae derived just before.

$$
\begin{array}{ll}
\Rightarrow & \tilde{v}_{e}=v_{e}+0.5 \gamma(\bar{c}-\nu) v_{e}=r_{1} v_{e}, \\
\Rightarrow & (1+0.5 \gamma \nu) \tilde{v}_{o}=v_{o}+0.5 r_{1} \gamma \bar{c} v_{o} \quad \Rightarrow \quad \tilde{v}_{o}=r_{2} v_{o}, \\
\Rightarrow & \hat{v}_{o}=r_{2} v_{o}+0.5 \gamma\left(r_{1} \bar{c}-r_{2} \nu\right) v_{o}=r_{3} v_{o}, \\
\Rightarrow & (1+0.5 \gamma \nu) \hat{v}_{e}=r_{1} v_{e}+0.5 r_{3} \gamma \bar{c} v_{e} \Rightarrow \hat{v}_{e}=r_{4} v_{e} .
\end{array}
$$

Analogously we can find transformations of function $w=v_{\mathbf{N}-\mathbf{k}}$ and define coefficients $r_{1}^{\prime}, r_{2}^{\prime}, r_{3}^{\prime}, r_{4}^{\prime}$. Thus, we have

$$
r_{1}=1-0.5 \gamma(\nu-\bar{c}), \quad r_{1}^{\prime}=1-0.5 \gamma(\nu+\bar{c}),
$$




$$
\begin{array}{ll}
r_{2}=\left(1+0.5 r_{1} \gamma \bar{c}\right) /(1+0.5 \gamma \nu), & r_{2}^{\prime}=\left(1-0.5 r_{1}^{\prime} \gamma \bar{c}\right) /(1+0.5 \gamma \nu), \\
r_{3}=r_{2}-0.5 \gamma\left(r_{2} \nu-r_{1} \bar{c}\right), & r_{3}^{\prime}=r_{2}^{\prime}-0.5 \gamma\left(r_{2}^{\prime} \nu-r_{1}^{\prime} \bar{c}\right), \\
r_{4}=\left(r_{1}+0.5 r_{3} \gamma \bar{c}\right) /(1+0.5 \gamma \nu), & r_{4}^{\prime}=\left(r_{1}^{\prime}-0.5 r_{3}^{\prime} \gamma \bar{c}\right) /(1+0.5 \gamma \nu) .
\end{array}
$$

Note that for the first boundary value problem $v_{e}=(-1)^{d} w_{e}, v_{o}=-(-1)^{d} w_{o}$ and for the second boundary value problem $v_{e}=w_{e}, v_{o}=-w_{o}$. Thus, for the first $(\kappa=1)$ and the second $(\kappa=2)$ boundary problems we have

$$
A v=\frac{r_{3}+r_{4}}{2} v+(-1)^{d \kappa} \frac{r_{4}-r_{3}}{2} w, \quad A w=(-1)^{d \kappa} \frac{r_{4}^{\prime}-r_{3}^{\prime}}{2} v+\frac{r_{3}^{\prime}+r_{4}^{\prime}}{2} w .
$$

If we introduce a linear operator $\tilde{A}, \tilde{p}=\tilde{A} p$, we can write analogous equalities for $\tilde{A} v$ and $\tilde{A} w$, where $r_{1}, r_{1}^{\prime}, r_{2}, r_{2}^{\prime}$ are instead of $r_{4}, r_{4}^{\prime}, r_{3}, r_{3}^{\prime}$ respectively. Operators $\tilde{A}$ and $A$ transform any linear combination of the functions $v$ and $w$ into a linear combination of these same functions. Thus, $v, w, \tilde{v}, \tilde{w}, \hat{v}, \hat{w}$ satisfy the boundary conditions.

As we have noticed in $\S 3$ using the functions $v_{\mathbf{k}}$ as a basis of a space $\mathbf{B}$ and numbering functions $v_{\mathbf{k}}$ and $v_{\mathbf{N}-\mathbf{k}}$ as a neighbouring vectors, we can write the operator $A$ as a blocked diagonal matrix which has $2 \times 2$ blocks $A_{\mathbf{k}}$ on a diagonal. Taking $v_{\mathbf{k}}=v$ and $v_{\mathbf{N}-\mathbf{k}}=w$, using the expressions of $r_{j}, r_{j}^{\prime}$ and considering $\kappa=1$ or $\kappa=2$ for the first or second boundary problems respectively, we have the following matrix $A_{\mathbf{k}}$ :

$$
A_{\mathbf{k}}=\frac{1}{2}\left(\begin{array}{cc}
\frac{(2+\gamma \bar{c})\left(4+\gamma^{2}\left(\bar{c}^{2}-\nu^{2}\right)+2 \gamma \bar{c}\right)}{(2+\gamma \nu)^{2}} & -(-1)^{d \kappa} \frac{\gamma^{3} \bar{c}(\bar{c}+\nu)^{2}}{(2+\gamma \nu)^{2}} \\
(-1)^{d \kappa} \frac{\gamma^{3} \bar{c}(\bar{c}-\nu)^{2}}{(2+\gamma \nu)^{2}} & \frac{(1-\gamma \bar{c})\left(4+\gamma^{2}\left(\bar{c}^{2}-\nu^{2}\right)-2 \gamma \bar{c}\right)}{(2+\gamma \nu)^{2}}
\end{array}\right) .
$$

It can also happen, that $v=w$ (when $\mathbf{k}=0.5 \mathbf{N}$ ) and instead of the pair of functions we have only one function. In this case $\bar{c}=0$ and operator $A$ changes $v$ in the odd and even grid points equivalently and the eigenvalue $q=r_{3}=r_{4}=(2-\gamma \nu) /(2+\gamma \nu),|q| \leq 1$.

Note, that in the cases of the second boundary problem and of the first boundary problem in even-dimensional space ( $d$ is even) function $v$ has zero values in all odd grid points. In the case of the first boundary problem in odd-dimensional space ( $d$ is odd) $v$ has zero values in all even grid points. Thus, one of the equalities $\tilde{v}_{o}=r_{2} v_{o}=r_{2} \cdot 0=r_{1} \cdot 0=r_{1} v_{o}$ or $\tilde{v}_{e}=r_{1} v_{e}=$ $r_{1} \cdot 0=r_{2} \cdot 0=r_{2} v_{e}$ is satisfied in any case and function $v$ (if it exists) is also the eigenfunction of the operator $\tilde{A}$. Therefore, $v, \tilde{v}$ and $\hat{v}$ satisfy the boundary conditions.

We shall check that for any other $\mathbf{k} \neq 0.5 \mathbf{N}$ the eigenvalues of the operator $A_{\mathbf{k}}$ also satisfy the condition $|q| \leq 1$ and, following definitions of $\S 3$, we shall conclude that the scheme is stable by means of von Neumann.

Let us write the characteristic equation for operator $A_{\mathbf{k}}$ :

$$
(2+\gamma \nu)^{2} q^{2}-2\left(2 \gamma^{2} \bar{c}^{2}-\gamma^{2} \nu^{2}+4\right) q+(2-\gamma \nu)^{2}=0 .
$$


Denoting

$$
\begin{gathered}
c=\bar{c} / \nu, \quad s^{2}=1-c^{2}, \quad 4-s^{2} \gamma^{2} \nu^{2}=D_{1}+i D_{2}, \quad D_{1}=4-\left(a^{2}-b^{2}\right) s^{2} \nu^{2}, \\
D_{2}=-2 a b s^{2} \nu^{2}, \quad D=\sqrt{D_{1}^{2}+D_{2}^{2}}=\sqrt{\left(4+|\gamma|^{2} s^{2} \nu^{2}\right)^{2}-16 a^{2} s^{2} \nu^{2}}
\end{gathered}
$$

we can write the following expression of $q$ :

$$
q=\frac{\left(2 \gamma^{2} \bar{c}^{2}-\gamma^{2} \nu^{2}+4\right) \pm 2 \gamma \bar{c} \sqrt{\gamma^{2} \bar{c}^{2}-\gamma^{2} \nu^{2}+4}}{(2+\gamma \nu)^{2}}=\left(\frac{c \gamma \nu \pm \sqrt{4-s^{2} \gamma^{2} \nu^{2}}}{2+\gamma \nu}\right)^{2}
$$

Now the stability condition $|q| \leq 1$ is equivalent to the inequality

$$
\left|c \gamma \nu \pm \sqrt{4-s^{2} \gamma^{2} \nu^{2}}\right|^{2} \leq|2+\gamma \nu|^{2}
$$

Using the formula of square root for complex number $D_{1}+i D_{2}$

$$
\sqrt{D_{1}+i D_{2}}= \pm\left(\sqrt{\left(D+D_{1}\right) / 2}+i \operatorname{sign}\left(D_{2}\right) \sqrt{\left(D-D_{1}\right) / 2}\right)
$$

where $\operatorname{sign}\left(D_{2}\right)= \pm 1$ if $D_{2} \geq 0$ or $D_{2}<0$ respectively, we can write

$\left|c \gamma \nu \pm \sqrt{4-s^{2} \gamma^{2} \nu^{2}}\right|^{2}=c^{2} \nu^{2}|\gamma|^{2}+D \pm c \nu \sqrt{2}\left(a \sqrt{D+D_{1}}+b \operatorname{sign}\left(D_{2}\right) \sqrt{D-D_{1}}\right)$.

Now the stability condition $|q| \leq 1$ reads as follows:

$$
\left(D-4-|\gamma|^{2} s^{2} \nu^{2}\right)+\nu\left(\sqrt{2 c^{2}}\left|a \sqrt{D+D_{1}}+b \operatorname{sign}\left(D_{2}\right) \sqrt{D-D_{1}}\right|-4 a\right) \leq 0 .
$$

Both terms at the left-hand side of this stability condition are not positive. Proving this statement we can write the equivalent inequalities for the first and the second terms at the left-hand side of the stability condition. Since

$$
\begin{gathered}
\left.D-4-|\gamma|^{2} s^{2} \nu^{2}\right) \leq 0 \quad \equiv-16 a^{2} s^{2} \nu^{2} \leq 0 \\
\sqrt{2 c^{2}}\left|a \sqrt{D+D_{1}}+b \operatorname{sign}\left(D_{2}\right) \sqrt{D-D_{1}}\right| \leq 4 a \equiv|c \gamma|^{2}\left(D-4-|s \nu \gamma|^{2}\right) \leq 8 a^{2} s^{2},
\end{gathered}
$$

the stability condition $|q| \leq 1$ for eigenvalues of the operator $A_{\mathbf{k}}$ as well as of the operator $A$ is satisfied.

THEOREM 7.1. Suppose that the solution of the differential problem is smooth enough. Then the difference scheme (7.1),(2.4),(2.5) unconditionally satisfies von Neumann's necessary stability condition for all evolutionary equations. 


\section{DUFORT-FRANKEL TYPE DIFFERENCE SCHEME}

We investigate an explicit three-layered finite difference scheme with weight $\sigma \in[0, \infty)$ :

$$
\frac{\hat{p}-\check{p}}{2 \tau}=(a+i b)\left(\Delta_{h} p+\sigma \sum_{k=1}^{d} \frac{2 p-\hat{p}-\check{p}}{h_{k}^{2}}\right), \quad(\mathbf{x}, t) \in Q_{h}, \quad\left(\text { or } \tilde{Q}_{h}\right) .
$$

We can write a scheme (8.1) as follows:

$$
\hat{p}=\frac{2 \gamma\left(\tau \Delta_{h}+\sigma \nu I\right)}{1+\gamma \sigma \nu} p+\frac{1-\gamma \sigma \nu}{1+\gamma \sigma \nu} \check{p} .
$$

The functions $v_{\mathbf{k}}$ from (2.7) or (2.8) are the eigenfunctions of the problem. Thus, $\check{p}, p, \hat{p}$ satisfy the boundary conditions. Using the notations (2.10) we can write an operator $A$ as a blocked diagonal matrix with the blocks $A_{\mathbf{k}}$ on a diagonal:

$$
A_{\mathbf{k}}=\left(\begin{array}{cc}
-\frac{2 \gamma \nu(2 \delta-\sigma)}{1+\gamma \sigma \nu} & \frac{1-\gamma \sigma \nu}{1+\gamma \sigma \nu} \\
1 & 0
\end{array}\right)
$$

The characteristic equation for operator $A_{\mathbf{k}}$ reads

$$
(1+\gamma \sigma \nu) q^{2}+2 \gamma \nu(2 \delta-\sigma) q-\left(1-\gamma^{2} \sigma^{2} \nu^{2}\right)=0 .
$$

For the simplicity of formulas, let us denote

$$
\begin{aligned}
& \left(1-4\left(a^{2}-b^{2}\right) \nu^{2} \delta(\sigma-\delta)\right)+i\left(-8 a b \nu^{2} \delta(\sigma-\delta)\right)=D_{1}+i D_{2}, \\
& D=\sqrt{D_{1}^{2}+D_{2}^{2}}=\sqrt{\left(1+4|\gamma|^{2} \nu^{2} \delta(\sigma-\delta)\right)^{2}-16 a^{2} \nu^{2} \delta(\sigma-\delta)} .
\end{aligned}
$$

Using (7.2) we can write the roots of the characteristic equation as follows:

$$
q=\frac{\left(a \nu(\sigma-2 \delta) \pm \sqrt{\left(D+D_{1}\right) / 2}\right)+i\left(b \nu(\sigma-2 \delta) \pm \operatorname{sign}\left(D_{2}\right) \sqrt{\left(D-D_{1}\right) / 2}\right)}{(1+a \sigma \nu)+i b \sigma \nu} .
$$

The stability condition $|q| \leq 1+C \tau$ is equivalent to the inequality

$$
\begin{aligned}
& \nu\left(\sqrt{2}\left|(\sigma-2 \delta)\left(a \sqrt{D+D_{1}}+b \operatorname{sign}\left(D_{2}\right) \sqrt{D-D_{1}}\right)\right|-2 a \sigma\right) \\
+\quad & \left(D-1-4|\gamma|^{2} \nu^{2} \delta(\sigma-\delta)\right) \leq C|1+\gamma \sigma \nu|^{2} \tau .
\end{aligned}
$$

Suppose that $\sigma-\delta \geq 0$. This condition can be achieved for all possible $\delta$ if only $\sigma \in[1 ; \infty)$. Analogously as in $\S 7$ both terms in the left-hand side of the last inequality are non-positive and, therefore, the estimate $|q| \leq 1$ is valid. 
Suppose now that $\sigma \in[0,1)$. For $\tau, h_{j}$ small enough we can always find $\lambda$ from (2.9) such that $\sigma<\delta=\lambda \tau /(2 \nu) \leq 1$. Particularly this estimate holds when we take $\delta=1$ for the second boundary problem and $1>\delta \rightarrow 1$ when $\tau, \bar{h} \rightarrow 0$ for the first boundary problem. Using standard investigation of the left-hand side of the stability inequality it is possible to find that it's biggest value is achieved when $1=\delta \in(\sigma ; 1]$. Now we can rewrite the stability condition as follows:

$$
\begin{aligned}
& \left(\left(D-D_{1}\right)+2 \nu(2-\sigma)\left(\sqrt{|\gamma|^{2}\left(D-D_{1}\right) / 2+4 a^{2}|\gamma|^{2} \nu^{2}(1-\sigma)+a^{2}}-a\right)\right) \\
+ & \left(8 a^{2} \nu^{2}(1-\sigma)+4 a \nu(1-\sigma)\right) \leq C\left(1+|\gamma|^{2} \sigma^{2} \nu^{2}+2 a \sigma \nu\right) \tau .
\end{aligned}
$$

Note, that both terms at the left-hand side of this inequality are nonnegative.

Let us consider $a>0$. If we suppose that the scheme is stable, the estimate

$8 a^{2} \nu^{2}(1-\sigma)+4 a \nu(1-\sigma) \leq C\left(1+|\gamma|^{2} \sigma^{2} \nu^{2}+2 a \sigma \nu\right) \tau \equiv \nu+\nu^{2} \leq C\left(1+\sigma^{2} \nu^{2}\right) \tau$

when $\tau, \bar{h} \rightarrow 0$ must be valid.

If $0<\nu \rightarrow 0$ it is equivalent to $\nu \leq C \tau$ and $1 \leq C h^{2}$.

If $0<C_{1} \leq \nu \leq C_{2}<\infty$, it is equivalent to $1 \leq C \tau$.

If $\nu \rightarrow \infty$, it is equivalent to $\nu^{2} \leq C \tau \nu^{2} \Rightarrow 1 \leq C \tau$, or, if $\sigma=0$, to $\nu^{2} \leq C \tau$.

It is clear that while $\tau, \bar{h} \rightarrow 0$ in all these cases the stability conditions can not be satisfied and the scheme (8.1) is unstable when $a>0$ and $\sigma \in[0,1)$.

Suppose that $a=0$. In this case $D_{2}=0$ and the stability condition reads as

$$
\left(D-D_{1}\right)+\sqrt{2}|b| \nu(2-\sigma) \sqrt{D-D_{1}} \leq C\left(1+b^{2} \sigma^{2} \nu^{2}\right) \tau .
$$

If we have $D_{1}=D \geq 0$, or $\nu^{2} \leq 1 /\left(4(1-\sigma) b^{2}\right)$, the left-hand side of this inequality is zero and the condition of stability is satisfied.

If $D_{1}=-D<0$, or $\nu^{2}>1 /\left(4(1-\sigma) b^{2}\right)$, then $\left(D-D_{1}\right)=2 D_{1}$ and now the stability condition is

$$
2 D+2|b| \nu(2-\sigma) \sqrt{D} \leq C\left(1+b^{2} \sigma^{2} \nu^{2}\right) \tau .
$$

If $\nu^{2} \rightarrow \infty$, then inserting the value of $D$ into the formula we obtain equivalent condition of stability $\nu^{2} \leq C \tau \nu^{2}$ and $1 \leq C \tau$. If $\sigma=0$, then we have $\nu^{2} \leq C \tau$. This inequality is not valid, when $\tau \rightarrow 0$.

If $1 /\left(4 b^{2}(1-\sigma)\right)<\nu^{2} \leq C_{1}<\infty$, then the stability condition is equivalent to $D+\sqrt{D} \leq C \tau$.

If $D \geq C_{2}>0$, the last estimate leads to $1 \leq C \tau$, that is not valid.

If $0<D \rightarrow 0$, we have $D+\sqrt{D} \leq C \tau \equiv D \leq C \tau^{2} \equiv 4 b^{2}(1-\sigma) \nu^{2} \leq$ $1+C \tau^{2}$.

Since $\nu^{2} \leq C_{1}$, the stability criterion can be written as

$$
4 b^{2}(1-\sigma) \nu^{2} \leq 1+C\left(\sum_{l=1}^{d} \frac{1}{h_{l}^{2}}\right)^{-2} \leq 1+C h^{4}, \quad \text { or } \quad \nu \leq \frac{1}{2|b| \sqrt{1-\sigma}}+C h^{4} .
$$


THEOREM 8.1. Suppose that the solution of the differential problem is smooth enough. Then if $\sigma \in[1, \infty)$, the difference scheme (8.1),(2.4),(2.5) (or (2.6)) is unconditionally stable for the evolutionary equation of any type by means of necessary von Neumann's stability condition.

In the case when $\sigma \in[0,1)$, this scheme is unconditionally unstable for the Kuramoto-Tsuzuki equations and is conditionally stable by means of von Neumann for Schrödinger equation if only the following condition is valid:

$$
\sum_{k=1}^{d} \frac{2 \tau}{h_{k}^{2}} \leq \frac{1}{2 \sqrt{b^{2}(1-\sigma)}}+C h^{4} .
$$

\section{THREE-LAYERED WEIGHTED DIFFERENCE SCHEME}

We consider a three-layered difference scheme with weight $\sigma \in[0, \infty)$ :

$$
\frac{\hat{p}-\check{p}}{2 \tau}=(a+i b) \Delta_{h}(\sigma \hat{p}+(1-2 \sigma) p+\sigma \check{p}), \quad(\mathbf{x}, t) \in Q_{h}, \quad\left(\text { or } \tilde{Q}_{h}\right) .
$$

Analogously as in $\S 8$ we write a scheme (9.1) as

$$
\hat{p}=2 \tau \gamma(1-2 \sigma)\left(I-2 \tau \gamma \sigma \Delta_{h}\right)^{-1} \Delta_{h} p+\left(I-2 \tau \gamma \sigma \Delta_{h}\right)^{-1}\left(I+2 \tau \gamma \sigma \Delta_{h}\right) \check{p} .
$$

The functions $v_{\mathbf{k}}$ from (2.7) or (2.8) are the eigenfunctions of the problem and the boundary conditions are satisfied for $\hat{p}, p$ and $\check{p}$. An operator $A$ can be written as a blocked diagonal matrix with blocks $A_{\mathbf{k}}$ on a diagonal and the characteristic equation for $A_{\mathbf{k}}$ is

$$
(1+4 \gamma \delta \nu \sigma) q^{2}+4 \gamma \delta \nu(1-2 \sigma) q-\left(1-16 \gamma^{2} \delta^{2} \nu^{2} \sigma^{2}\right)=0 .
$$

Once again we introduce the notations

$$
\begin{aligned}
& \left(1+4\left(a^{2}-b^{2}\right) \delta^{2} \nu^{2}(1-4 \sigma)\right)+i\left(8 a b \delta^{2} \nu^{2}(1-4 \sigma)\right)=D_{1}+i D_{2}, \\
& D=\sqrt{D_{1}^{2}+D_{2}^{2}}=\sqrt{\left(1-4|\gamma|^{2} \delta^{2} \nu^{2}(1-4 \sigma)\right)^{2}+16 a^{2} \delta^{2} \nu^{2}(1-4 \sigma)} .
\end{aligned}
$$

Using these notations and (7.2), analogously as in $\S 8$ we can write the the stability inequality equivalent to the estimate $|q| \leq 1+C \tau$ :

$$
\begin{aligned}
& 2 \delta \nu\left(\sqrt{2}\left|(1-2 \sigma)\left(a \sqrt{D+D_{1}}+b \operatorname{sign}\left(D_{2}\right) \sqrt{D-D_{1}}\right)\right|-4 a \sigma\right) \\
+\quad & \left(D-1+4|\gamma|^{2} \delta^{2} \nu^{2}(1-4 \sigma)\right) \leq C|1+4 \gamma \delta \nu \sigma|^{2} \tau .
\end{aligned}
$$

When $\sigma \in[0.25, \infty)$, we have $1-4 \sigma \leq 0$ and analogously as earlier we can obtain non-positiveness of the left-hand side of the stability inequality. 
If $\sigma \in[0,0.25)$, then, noting that the left-hand side of the stability inequality is biggest when $1=\delta \in[0 ; 1]$, we rewrite the stability condition as

$$
\begin{aligned}
& \left(\left(D-D_{1}\right)+4 \nu(1-2 \sigma)\left(\sqrt{|\gamma|^{2}\left(D-D_{1}\right) / 2+4 a^{2}|\gamma|^{2} \nu^{2}(1-4 \sigma)+a^{2}}-a\right)\right) \\
& +\left(8 a^{2} \nu^{2}(1-4 \sigma)+4 a \nu(1-4 \sigma)\right) \leq C|1+4 \gamma \sigma \nu|^{2} \tau .
\end{aligned}
$$

If $a>0$, analogously as in $\S 8$ we show that the estimate

$8 a^{2} \nu^{2}(1-4 \sigma)+4 a \nu(1-4 \sigma) \leq C|1+4 \gamma \sigma \nu|^{2} \tau \equiv \nu+\nu^{2} \leq C\left(1+\sigma^{2} \nu^{2}\right) \tau$

can not be satisfied when $\tau, \bar{h} \rightarrow 0$ and the scheme is not stable.

If $a=0$, the stability condition reads as

$$
\left(D-D_{1}\right)+4|b| \nu(1-2 \sigma) \sqrt{\left(D-D_{1}\right) / 2} \leq C\left(1+16 b^{2} \sigma^{2} \nu^{2}\right) \tau
$$

and this condition is satisfied if $\nu \leq 1 /(2|b| \sqrt{1-4 \sigma})+C h^{4}$.

THEOREM 9.1. Suppose that the solution of the differential problem is smooth enough. Then if $\sigma \in[0.25, \infty)$, the difference scheme (9.1),(2.4),(2.5) (or (2.6)) is unconditionally stable for the evolutionary equation of any type by means of necessary von Neumann's stability condition.

In the case when $\sigma \in[0,0.25)$, this scheme is unconditionally unstable for the Kuramoto-Tsuzuki equations and is conditionally stable by means of von Neumann for Schrödinger equation if only the following condition is valid:

$$
\sum_{k=1}^{d} \frac{2 \tau}{h_{k}^{2}} \leq \frac{1}{2 \sqrt{b^{2}(1-4 \sigma)}}+C h^{4} .
$$

\section{CONCLUSIONS}

We use the following difference schemes to approximate the problem (2.1-2.3):

1. Two-layered weighted difference schemes (4.1);

2. Weighted splitting scheme I (5.1);

3. Weighted splitting scheme II (6.1);

4. Hopscotch scheme (7.1);

5. DuFort-Frankel type weighted schemes (8.1);

6. Three-layered weighted schemes (9.1).

The necessary von Neumann's conditions of the stability on initial data for these schemes depend on the real constants $a$ and $b$ of the equation (2.1) and on parameters of the difference schemes. All these conditions are presented in the table.

Looking at this table one can notice that in the case of 1,2 , and 3 scheme, the necessary von Neumann's conditions are also sufficient. In these cases the 


\begin{tabular}{|c|c|c|c|c|}
\hline \multirow{2}{*}{\multicolumn{2}{|c|}{ Method }} & \multirow{2}{*}{$\begin{array}{l}\text { Truncation } \\
\text { error }\end{array}$} & \multicolumn{2}{|c|}{ Stability conditions } \\
\hline & & & $a>0, b=0$ or $a>0, b \neq 0$ & $a=0, b \neq 0$ \\
\hline 1 & $\begin{array}{l}\sigma \in[0 ; 0.5) \\
\sigma=0.5 \\
\sigma \in(0.5 ; \infty)\end{array}$ & $\begin{array}{l}\bar{h}^{2}+\tau \\
\bar{h}^{2}+\tau^{2} \\
\bar{h}^{2}+\tau\end{array}$ & $\begin{array}{l}\sum \frac{2 \tau}{h_{k}^{2}} \leq \frac{a}{\left(a^{2}+b^{2}\right)(1-2 \sigma)}+C h^{2} \\
\text { Stable } \\
\text { Stable }\end{array}$ & $\begin{array}{l}\sum \frac{\tau}{h_{k}^{4}} \leq C \\
\text { Stable } \\
\text { Stable }\end{array}$ \\
\hline 2 & $\begin{array}{l}\exists k \sigma_{k} \in[0 ; 0.5) \\
\forall k \sigma_{k}=0.5 \\
\forall k \sigma_{k} \in(0.5 ; \infty)\end{array}$ & $\begin{array}{l}\bar{h}^{2}+\tau \\
\bar{h}^{2}+\tau^{2} \\
\bar{h}^{2}+\tau\end{array}$ & $\begin{array}{l}\frac{2 \tau}{h_{k}^{2}} \leq \frac{a}{\left(a^{2}+b^{2}\right)\left(1-2 \sigma_{k}\right)}+C h_{k}^{2} \\
\text { Stable } \\
\text { Stable }\end{array}$ & $\begin{array}{l}\frac{\tau}{h_{k}^{4}} \leq C \\
\text { Stable } \\
\text { Stable }\end{array}$ \\
\hline 3 & $\begin{array}{l}\sum \frac{\alpha_{k}}{\sigma_{k}} \in(0 ; 2] \\
\sum \frac{\alpha_{k}}{\sigma_{k}} \in(2 ; \infty)\end{array}$ & $\begin{array}{l}\bar{h}^{2}+\tau \\
\bar{h}^{2}+\tau\end{array}$ & $\begin{array}{l}\text { Stable } \\
\forall k \frac{2 \tau}{h_{k}^{2}} \leq \frac{a \alpha_{k}}{\varepsilon\left(a^{2}+b^{2}\right) \sigma_{k}}+C h_{k}^{2}\end{array}$ & $\begin{array}{l}\text { Stable } \\
\forall k \frac{\tau}{h_{k}^{2}} \leq C\end{array}$ \\
\hline 4 & & $\bar{h}^{2}+\tau^{2}$ & Stable* & Stable * \\
\hline 5 & $\begin{array}{l}\sigma=0 \\
\sigma \in(0 ; 1) \\
\sigma \in[1 ; \infty)\end{array}$ & $\begin{array}{l}\bar{h}^{2}+\tau^{2} \\
\bar{h}^{2}+\tau^{2}+\left(\frac{\tau}{h}\right) \\
\bar{h}^{2}+\tau^{2}+\left(\frac{\tau}{h}\right)\end{array}$ & $\begin{array}{l}\text { Unstable } \\
\text { Unstable } \\
\text { Stable* }\end{array}$ & $\begin{array}{l}\sum \frac{2 \tau}{h_{k}^{2}} \leq \frac{1}{2|b|}+C h^{4} * \\
\sum \frac{2 \tau}{h_{k}^{2}} \leq \frac{1}{2|b| \sqrt{1-\sigma}}+C h^{4 *} \\
\text { Stable* }\end{array}$ \\
\hline 6 & $\begin{array}{l}\sigma \in[0 ; 0.25) \\
\sigma \in[0.25 ; \infty)\end{array}$ & $\begin{array}{l}\bar{h}^{2}+\tau^{2} \\
\bar{h}^{2}+\tau^{2}\end{array}$ & $\begin{array}{l}\text { Unstable } \\
\text { Stable * }\end{array}$ & $\begin{array}{l}\sum \frac{2 \tau}{h_{k}^{2}} \leq \frac{1}{2|b| \sqrt{1-4 \sigma}}+C h^{4 *} \\
\text { Stable* }\end{array}$ \\
\hline
\end{tabular}

- It is sufficient condition. $\varepsilon=-2+\sum \frac{\alpha_{k}}{\sigma_{k}}$.

* Stability by mens of von Neumann's necessary condition

stability conditions continuously depend on the real constants $a$ and $b$. For the 3-rd scheme this condition depends on too much parameters, thus we have written only a sufficient stability condition.

Note also, that 4, 5 and 6 difference schemes were investigated only by means of necessary von Neumann's stability criterion. It is also interesting to note that 5 and 6 finite difference schemes with corresponding parameters $\sigma$ are unconditionally unstable if $a>0$, but they can be stable if $a=0$.

\section{REFERENCES}

[1] Čiegis R., Štikoniene O.: Explicit schemes for nonlinear Schrödinger type equations, Report of Lithuanian Inst. of Math. and Informatics, 97-13, 1997.

[2] Dai W.: Absolute stable explicit and semi-explicit schemes for Schrödinger equations, Math. Numer. Sinica, 11, 128-131, 1989.

[3] Fei Z., Perez-Garcia V., Vazquez L.: Numerical Simulation of Nonlinear Schrödinger Systems: A New Conservative Scheme, Appl. Math. Comput., 71, 165-177, 1995.

[4] Ivanauskas F.: Convergence and Stability of Difference Schemes for Nonlinear Schrödinger Equations, the Kuramoto-Tsuzuki Equation and Systems of ReactionDiffusion Type, Russian Acad. Sci. Dokl. Math., 50, 122-126, 1995.

[5] Morton K.W.: Stability of finite difference approximations to a diffusion-convection equation, Intern. J. Numer. Methods Engrg., 15, 677-683, 1980. 
[6] Richtmyer R.D., Morton K.W.: Difference Methods for Initial Value Problems, Interscience, New York, 1967.

[7] Samarskii A.A.: Theory of Difference Schemes [in Russian], Nauka, Moscow, 1989.

[8] Taha T.R., Ablowitz M.J.: Analytical and Numerical Aspects of Certain Nonlinear Evolution Equations. II. Numerical, Nonlinear Schrödinger Equation, J. Comput. Phys., 55, 203-230, 1984.

[9] ten Thije Boonkkamp J.H.M., Verwer J.G.: On the odd-even hopscotch scheme for the numerical integration of time-dependent partial differential equations, Appl. Numer. Math., 3, 183-193, 1987.

[10] Zaitseva S.B., Zlotnik A.A.: Optimal error estimates of one local one-dimensional method for multidimensional heat equation [in Russian], Mat. Zametki, 60, No.2, 185197, 1996. 